\title{
An Efficient Method for Vehicle License Plate Detection in Complex Scenes
}

\author{
Mahmood Ashoori-Lalimi, Sedigheh Ghofrani* \\ Electrical Engineering Department, Islamic Azad University, South Tehran Branch, Tehran, Iran \\ E-mail:mahmood_ashoori@yahoo.com, ${ }^{*}$ s_ghofrani@azad.ac.ir \\ Received April 2, 2011; revised August 23, 2011; accepted August 30, 2011
}

\begin{abstract}
In this paper, we propose an efficient method for license plate localization in the images with various situations and complex background. At the first, in order to reduce problems such as low quality and low contrast in the vehicle images, image contrast is enhanced by the two different methods and the best for following is selected. At the second part, vertical edges of the enhanced image are extracted by sobel mask. Then the most of the noise and background edges are removed by an effective algorithm. The output of this stage is given to a morphological filtering to extract the candidate regions and finally we use several geometrical features such as area of the regions, aspect ratio and edge density to eliminate the non-plate regions and segment the plate from the input car image. This method is performed on some real images that have been captured at the different imaging conditions. The appropriate experimental results show that our proposed method is nearly independent to environmental conditions such as lightening, camera angles and camera distance from the automobile, and license plate rotation.
\end{abstract}

Keywords: License Plate Detection, Image Enhancement, Background and Noise Removing, Morphological Operations

\section{Introduction}

With the rapid development of highway and the wide use of vehicle, researchers start to pay more attention on efficient and accurate intelligent transportation systems (ITS). It is widely used for detecting car's speed, security control in restricted areas, highway surveillance and electric toll collection [1]. Vehicle license plate (VLP) recognition is one of the most important requirements of an ITS. Although any ITS and specifically any VLP recognition contains two part in general, license plate detection and recognition, detecting and segmenting VLP correctly is most important because of existing conditions such as poor illumination, vehicle motion, viewpoint and distance changes. The problem of automatic VLP recognition has been studied since 1990s. The first approach was based on characteristics of boundaries $[2,3]$. In this method, an image was binarized and then processed by certain algorithms, such as Hough transform, to detect lines. In general, the most common approaches for VLP detection include texture [1,4], color feature [5], edge extraction [6], combining edge and color
[7], morphological operation [5,8] and learning-based method [9]. Using color feature is benefit when lightening is unchanged and stable. However methods based on edge and texture are nearly invariant to different illumination and so they are widely used for VLP detection. These methods use the fact that there are many characters in the license plate, so the area contains rich edge and texture information. Zhang et al. [9] proposed learning-based method using AdaBoost for VLP detection. They used both global (statistical) and local (Haar-like) features to detect the license plate.

In this paper, we do pre processing for image enhancement at first. The some regions are candidate as a license plate during three procedures. Finally considering geometrical features, the license plate is segmented nearly independent of image capturing conditions.

This paper is organized as follows: in Section 2, different styles of Iranian license plates are illustrated in Section 3. We express how our image bank is provided. In Section 4, the proposed algorithm is described and in Section 5 the experimental results are reported. Finally, in Section 6 we have conclusion. 


\section{Iranian Vehicle License Plate}

We have considered 3 classes for Iranian VLP, they are private, public (such as taxi, truck and bus) and governmental vehicles. Each class has own plate and character color. In addition, though Farsi characters are 32, only some characters are used for VLP. Color arrangement, characters and outline of the Iranian VLP are shown in Table 1.

\section{Provided Image Bank}

As respects, the aim of this paper is detecting the Iranian license plates in images with complex scenes. Due to the unavailability of required images, in several stages by using 2 digital cameras and mobile cameras, we have provided 350 images under various illumination (lightening), different distances and angles of stationary and moving vehicles. After providing images, in order to increase the processing speed and facilitate the license plate detection, input color image is converted to grayscale image. The size of images is $640 \times 480$ pixels.

\section{Efficient License Plate Detection}

Our proposed method is composed of several parts, Figure 1 shows the flowchart.

\subsection{Pre Processing}

Low contrast may have the most important effect on failing a license plate detection algorithm. Severe lightening conditions, changing plate orientation and various distances are main reasons for having low contrast and quality in the car images. Therefore, contrast enhancement seems to be necessary, specially at locations where might be a license plate. So, in following we improve different images using two methods, they are intensity variance [6] and edge density [7], and choose the best for pre processing images.

\subsubsection{Intensity Variance}

Zheng et al. [6] used the local variance of pixel intensities to improve image contrast at regions that may be plate. They proposed an enhancement function which increases image contrast at regions that local variance of intensity is around 20. The enhancement function was suggested as follows:

$$
I_{i j}^{\prime}=f\left(\sigma_{w_{i j}}\right)\left(I_{i j}-\bar{I}_{w_{i j}}\right)+\bar{I}_{w_{i j}}
$$

where $I_{i j}$, and $I_{i j}^{\prime}$ denote the intensities of the pixel in the input grayscale image and enhanced image, and $w_{i j}$ is a window centered on pixels of grayscale image. $\bar{I}_{w_{i j}}$, and $\sigma_{w_{i j}}$ are average luminance and standard deviation respectively. The enhanced coefficient is defined as follows:

$$
f\left(\sigma_{w_{i j}}\right)=\left\{\begin{array}{lll}
\frac{3}{\frac{2}{400}\left(\sigma_{w_{i j}}-20\right)^{2}+1} & \text { if } & 0 \leq \sigma_{w_{i j}}<20 \\
\frac{3}{\frac{2}{1600}\left(\sigma_{w_{i j}}-20\right)^{2}+1} & \text { if } & 20 \leq \sigma_{w_{i j}}<60 \\
1 & \text { if } \quad \sigma_{w_{i j}} \geq 60
\end{array}\right.
$$

With respect to Figure 2, the intensities of pixels in the input grayscale images with local variance between 0 and 60 are enhanced.

Figure 3 shows the result of image enhancement using the zheng's method.

\subsubsection{Edge Density}

Abolghasemi et al. [7] used the density of vertical edges (instead of the variance of intensity) as criterion for local enhancement of car image. License plate of the car consist of several characters (8 characters for Iranian VLP), so the license plate area contains rich edge information. We can employ the edge information to find the location of plate in an image. At first, they [7] used the vertical sobel mask and obtained the gradient image:

Table 1. Different styles of Iranian VLP.

\begin{tabular}{cccc}
\hline Vehicle Type & Plate Color & Character Color & Outline of VLP \\
\hline Private (automobile) & white & black & \\
Public (taxi, truck and bus) & yellow & black & \\
Governmental & red & white & \\
\hline
\end{tabular}




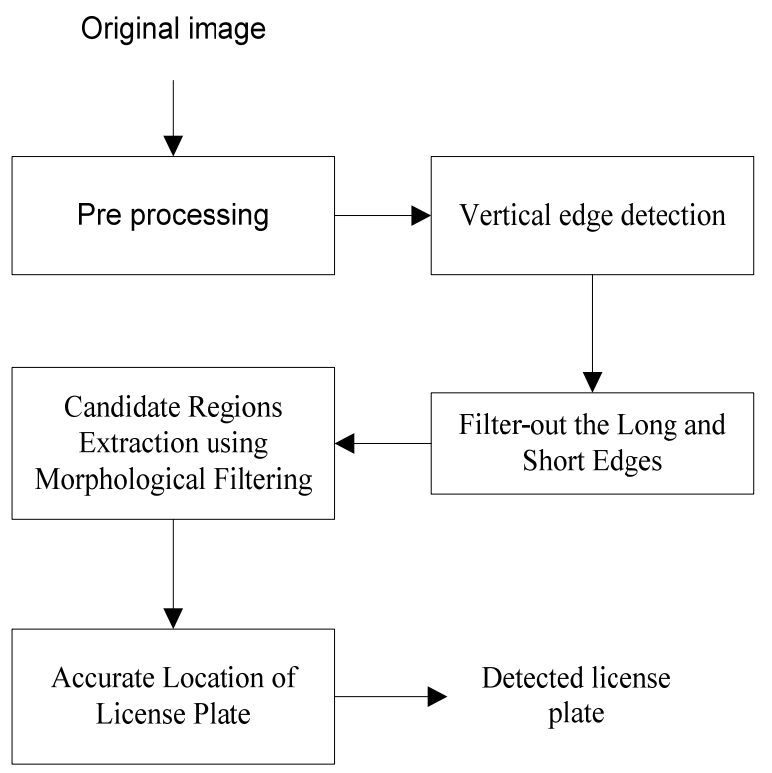

Figure 1. Flowchart of our proposed system for VLP detection.

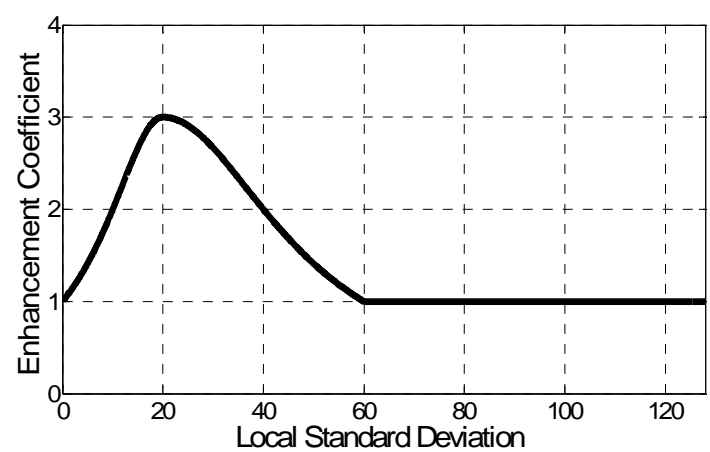

Figure 2. The graph of enhancement coefficient, $f\left(\sigma_{w_{i j}}\right)$, based on the local standard deviation, $\sigma_{w_{i j}}$.

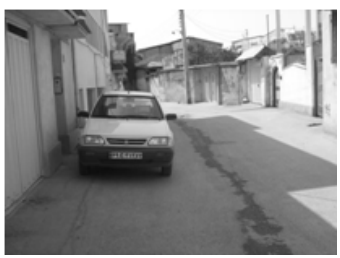

GAEYYSP (b)

\section{SQEYYYS (c)}

(a)

Figure 3. (a) input grayscale image. Iranian license plate before (b) and after (c) enhancement using intensity variance method.

$$
h=\left[\begin{array}{lll}
-1 & 0 & 1 \\
-2 & 0 & 2 \\
-1 & 0 & 1
\end{array}\right]
$$

Then, they compared pixel values with a predefined threshold and the vertical edge image has been achieved.
In the next step, the vertical edge image is convolved with the 2-D Gaussian kernel and estimation of the edge density is yielded. The results on a sample image are shown in Figure 4.

In order to enhance the input image with respect to the estimations of edge density, an enhancement coefficient is suggested as follows:

$$
I_{i j}^{\prime}=f\left(\rho_{w_{i j}}\right)\left(I_{i j}-\bar{I}_{w_{i j}}\right)+\bar{I}_{w_{i j}}
$$

where $I_{i j}, I_{i j}^{\prime}$ and $\bar{I}_{w_{i j}}$ are explained in the previous step. $f\left(\rho_{w_{i j}}\right)$ is the weighting function, regarding the estimation of edge density. This function is sketched in

\section{Figure 5.}

As can be seen in Figure 5, the intensity of pixels with the edge density among 0.15 to 0.45 is to be enhanced. The enhancement coefficient $f\left(\rho_{w_{i j}}\right)$ is defined as follows:

$$
\begin{aligned}
& f\left(\rho_{w_{i j}}\right) \\
& =\left\{\begin{array}{l}
\frac{3}{\frac{2}{0.15^{2}}\left(\rho_{w_{i j}}-0.15\right)^{2}+1}, \text { if } 0 \leq \rho_{w_{i j}}<0.15 \\
\frac{3}{\frac{2}{(0.5-0.15)^{2}}\left(\rho_{w_{i j}}-0.15\right)^{2}+1}, \text { if } 0.15 \leq \sigma_{w_{i j}}<0.5
\end{array}\right. \\
& 1, \quad \text { if } \rho_{w_{i j}} \geq 0.5
\end{aligned}
$$

Figure 6 shows the result of enhancement by this method.

Even though for normal images, as it can be seen in Figure 7(a), both intensity variance [6] and edge density [7] can improve the VLP, when the distance and angle

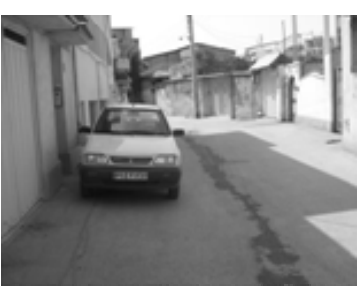

(a)

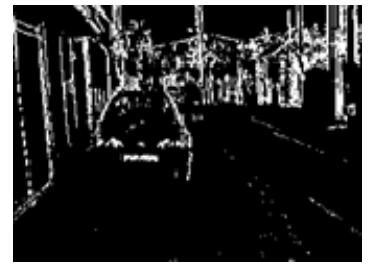

(b)

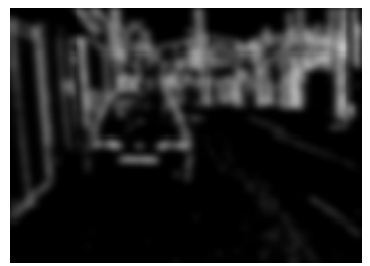

(c)
Figure 4. (a) Input grayscale image, (b) vertical edge image and (c) the edge-density estimation image. 


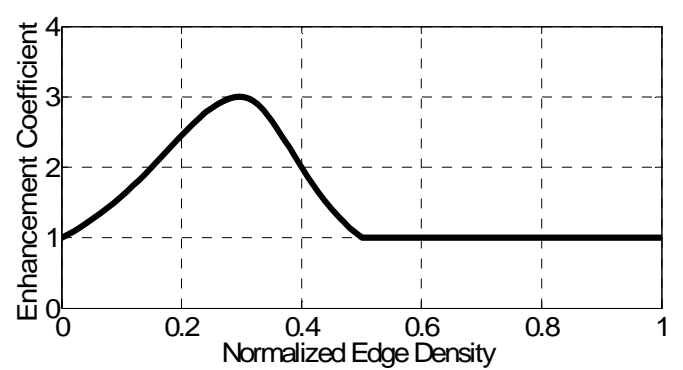

Figure 5. The graph of enhancement coefficient, $\boldsymbol{f}\left(\rho_{w_{i j}}\right)$, based on normalized edge density, $\rho_{w_{i j}}$.

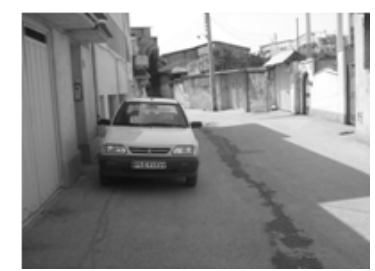

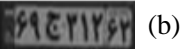

\section{GETाY (c)}

(a)

Figure 6. (a) input grayscale image. Iranian license plate before (b) and after (c) enhancement using edge density method.
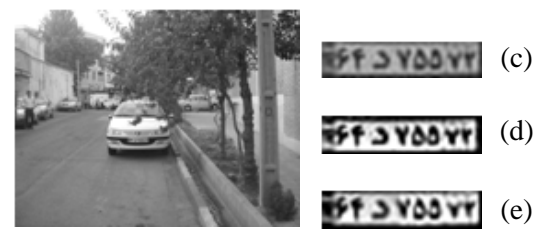

(a)
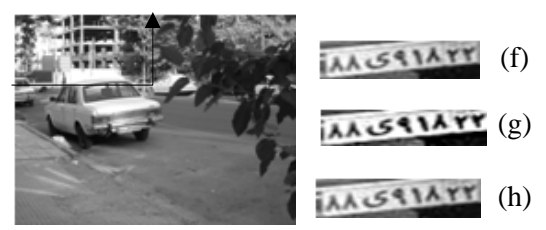

(b)

Figure 7. (a),(b) input grayscale image. Iranian license plate (c),(f) without enhancement (d),(g) improved using edge density method (e), (h) improved using intensity variance method.

between camera and vehicle are increased, zheng's method fails while abolghasemi's method already improves the quality of VLP considerably, Figure 7(b). so, in this work we employ edge density method for pre processing.

\subsection{Detecting the VLP}

After enhancing an input image by using suitable method (edge density), we should detect any existed license plate in the improved image. We do the following stages for this purpose.

\subsubsection{Vertical Edge Detection}

Edge detection is one of the most important processes in image analysis. An edge represents the boundary of an object which can be used to identify the shapes and area of the particular object. When there is contrast difference between the object and the background, after applying edge detection, the object edges will be illustrated. We select the vertical sobel operator, Equation (3), to detect the vertical edges.

After convolving the enhanced car image with the vertical sobel operator, an estimation of vertical gradient image is yielded. Finally, we get a binary image, as shown in Figure 8(a), by using a threshold value.

\subsubsection{Filter-Out the Long and Short Edges}

After extracting vertical edges from the enhanced image, using morphological filtering obtains candidate regions those may be a license plate. But, as it can be seen in Figure 8(a), there are many long background and short noise edges that may interference in the morphological filtering process. In order to resolve this problem, an effective algorithm is used to remove the background and noise edges [6]. The filter-out image after removing unwanted edges is shown in the Figure 8(b).

\subsubsection{Candidate Regions Extraction Using Morphological Filtering}

Morphological filtering is used as a tool for extracting image components and so representing and describing region shapes such as boundaries. In this part, we use a morphological operation for extracting candidate regions. Hence, we implement the morphological closing and opening that defined as follows:

$$
\begin{aligned}
& \text { Closing operation } I \bullet S_{m \times n}=\left(I \oplus S_{m \times n}\right) \Theta S_{m \times n} \\
& \text { Opening operation } I \circ S_{m \times n}=\left(I \Theta S_{m \times n}\right) \oplus S_{m \times n}
\end{aligned}
$$

where $\oplus$, and $\Theta$ denote dilation and erosion operations, respectively. $S_{m \times n}$ denote a structuring element with size $m \times n$, all entries in $S_{m \times n}$ are one. The output of this stage is shown in Figure 9(a).

\subsubsection{Accurate Location of License Plate}

After using morphological filtering, still many regions

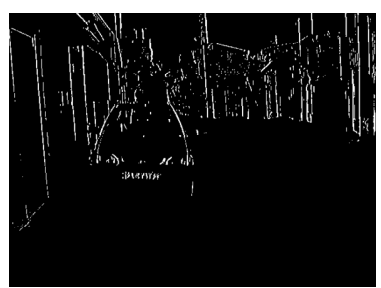

(a)

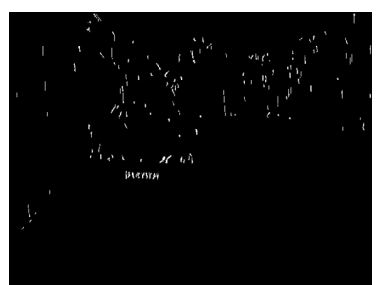

(b)
Figure 8. (a) vertical edge image, (b) removing background and noise edges (filter-out the long and short edges). 


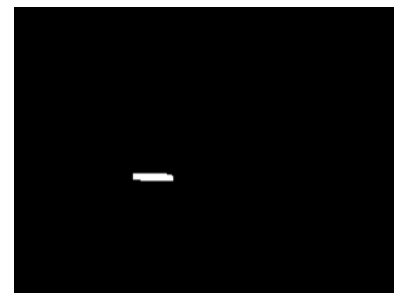

(a)

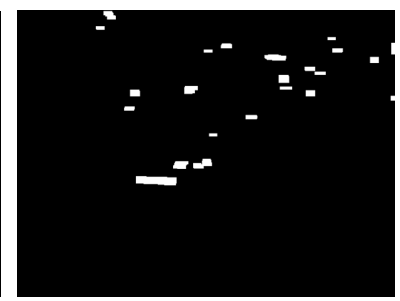

(b)

\section{Merips}

(c)

Figure 9. (a) Connected regions obtained from morphological process, (b) after applying features (c) cropped image.

are candidating as a license plate. So we consider some features such as area, aspect ratio (height per width) and edge density in order to discard wrong candidate regions. Values for these features are set experimentally based on our test images. These features are scale-, luminanceand rotation-variant. Progressive of using these features to remove non-plate candidate regions can be seen in Figure 9(b).

\section{Experimental Results}

We have run our proposed algorithm on laptop Core 2 Due CPU 2.26 MHz with $2 \mathrm{~GB}$ of RAM under MATLAB R2008b environment. In Section 3, we described how the vehicle images are provided. Some sample images of our database are shown in Figure 10.

Now, in order to evaluate the accuracy of our proposed method, we categorize the provided database into three categories including: Angle (high angle (>30 degree $\&<60$ degree) and low angle ( $<30$ degree)), Distance (short distance $(<4 \mathrm{~m})$ and normal distance $(>4 \mathrm{~m}$ $\&<12 \mathrm{~m}$ ) and low quality or contrast (evening, sunlight,

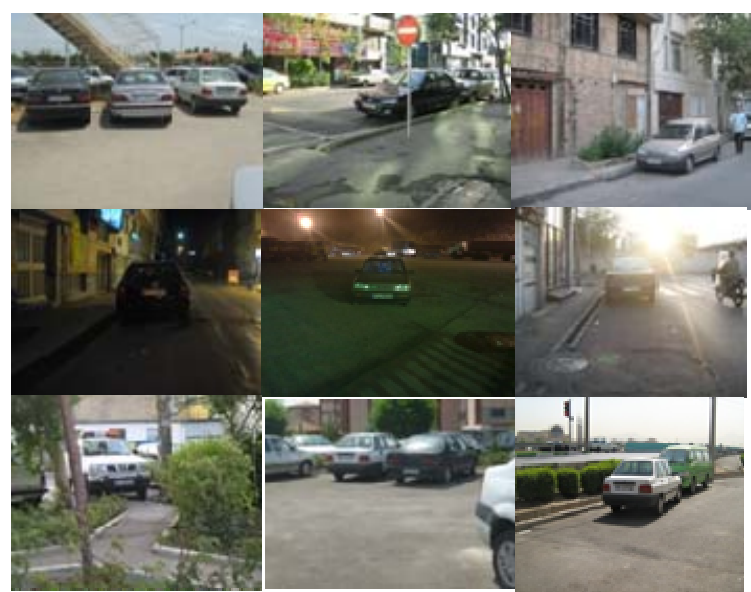

Figure 10. Some sample images of our database. and rainy or cloudy weather).

Table 2 shows the accuracy of the proposed algorithm under mentioned conditions.

As it is written in Table 2, the average accuracy achieved by our proposed method for license plate detection is $95.2 \%$. It means that we could detect 333 Iranian license plate correctly. Although our achieved accuracy is less than what zheng [6] got but we believe our provided image bank is more complex. Because of showing in Figure 7, the zheng's method is sensitive to real situations such as existing long distance and high angle. Table 3 shows the result of license plate detection for several vehicle images in our categories.

Table 2. Accuracy achieved by our proposed method.

\begin{tabular}{lccc}
\hline \multicolumn{2}{c}{$\begin{array}{c}\text { Images in Different } \\
\text { Situations }\end{array}$} & $\begin{array}{c}\text { Number of } \\
\text { Images }\end{array}$ & Accuracy (\%) \\
\hline \multirow{2}{*}{ Distance } & short & 100 & 97 \\
& normal & 100 & 95 \\
\multirow{2}{*}{ Angle } & low & 50 & 98 \\
& high & 50 & 94 \\
\multicolumn{2}{c}{ Low Quality } & 50 & 92 \\
& Average (350 Images) & & 95.2 \\
\hline
\end{tabular}

Table 3. Detected license plate by our proposed algorithm.

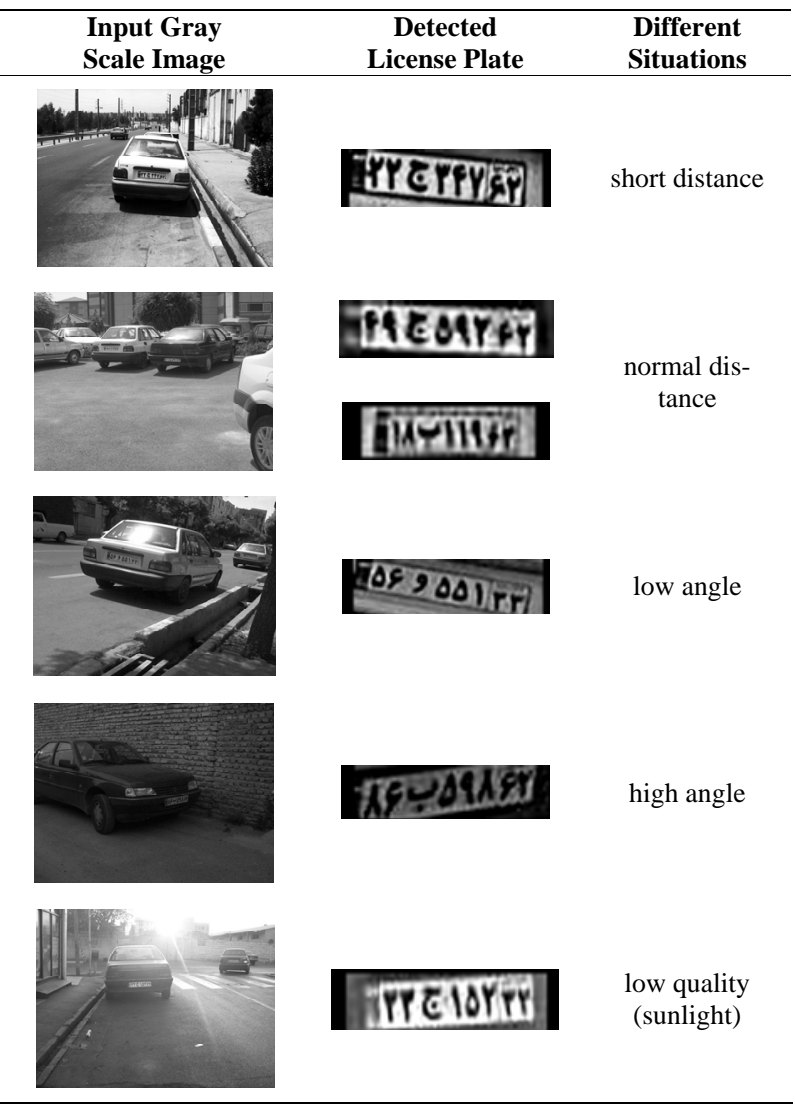




\section{Conclusions}

In this paper, an efficient license plate detection method is proposed which performed on images with complex scenes. We used edge density as pre processing for image enhancement. Then by using vertical sobel mask, removing background and noise edges, and employing morphological filter, some regions are candidate as license plate. Finally considering geometrical features (such as area of the regions, aspect ratio and edge density), the license plate from the input car image is extracted. Although the proposed algorithm performs on the Iranian vehicle license plates under various situations such as different lightening conditions, varied distances and existence angle between the camera and the vehicle and varied weather conditions, we believe its performance is yet appropriate if we try to detect the foreign license plate.

\section{References}

[1] V. Shapiro, G. Gluhchev and D. Dimov, "Toward a Multinational Car License Plate Recognition System,” Machine Vision Application, Vol. 17, No. 3, July 2006, pp. 173-183. doi:10.1007/s00138-006-0023-5

[2] V. Kamat and S. Ganesan, “An Efficient Implementation of the Hough Transform for Detecting Vehicle License Plates Using DSP's," Proceedings of Real Time Technology and Applications, Chicago, 15-17 May 1995, pp. 58-59. doi:10.1109/RTTAS.1995.516201
[3] Y. Yanamura, M. Goto and D. Nishiyama, "Extraction and Tracking of the License Plate Using Hough Transform and Voted Block Matching," IEEE proceedings of Intelligent Vehicles Symposium, Columbus, 9-11 June 2003, pp. 243-246.

[4] C. N. E. Anagnostopoulos and I. E. Anagnostopoulos, “A License Plate Recognition Algorithm for Intelligent Transportation System Applications," IEEE Transaction on Intelligent Transportation System, Vol. 7, No. 3, 2006, pp. 377-392.

[5] W. G. Zhu, G. J. Huo and X. Jia, “A Study of Locating Vehicle License Plate Based on Color Feature and Mathematical Morphology," 6th International Conference on Signal Processing, Vol. 1, 2002, pp. 748-751.

[6] D. Zheng, Y. Zhao and J. Wang, “An Efficient Method of License Plate Location,” Pattern Recognition Letters, Vol. 25, No. 6, 2005, pp. 2431-2438. doi:10.1016/j.patrec.2005.04.014

[7] V. Abolghasemi and A. R. Ahmadyfard, "An Edge-Based Color-Aided Method for License Plate Detection,” Elsevier Journal of Image and Vision Computing, Vol. 27 No. 8, 2009, pp. 1134-1142.

[8] J. W. Hsieh, S. H. Yu and Y. S. Chen, "MorphologyBased License Plate Detection from Complex Scenes," 16 th International Conference on Pattern Recognition, Vol. 3, 2002, pp. 176-179.

[9] H. Zhang, W. Jia, X. He and Q. Wu, "Learning-Based License Plate Detection Using Global and Local Features,” ICPR, Vol. 2, 2006, pp. 1102-1105. 